\title{
Fatty acid oxidation disorders as primary cause of sudden and unexpected death in infants and young children: an investigation performed on cultured fibroblasts from 79 children who died aged between $0-4$ years
}

\author{
J B Lundemose, S Kølvraa, N Gregersen, E Christensen, M Gregersen
}

Institute of Forensic Medicine, University of Aarhus,

Finsensgade 15, 8000

Aarhus C, Denmark

$\mathrm{J}$ B Lundemose

$M$ Gregersen

Institute of Human

Genetics

S Kølvraa

Center for Medical Molecular Biology,

Aarhus University

Hospital and Faculty of

Health Sciences,

Skejby Sygehus 8200

Aarhus N, Denmark

N Gregersen

Department of Clinical

Genetics,

Rigshospitalet,

Copenhagen, Denmark

E Christensen

Correspondence to:

Dr Lundemose.

Accepted for publication 29 May 1997

\begin{abstract}
Background-Disorders of fatty acid metabolism are known to be responsible for cases of sudden and unexpected death in infancy. At least 14 disorders are known at present. 120 cases of sudden infant death syndrome (SIDS) had been examined for a prevalent mutation (G985) causing medium chain acyl CoA dehydrogenase deficiency, which is inherited in an autosomal recessive mode. No over-representation of either homozygous or heterozygous cases was found.
\end{abstract}

Aims-To investigate a broader spectrum of fatty acid oxidation disorders in a wider range of sudden deaths in infants and young children.

Methods-Seventy nine cases of unexpected death in infants and young children younger than 4 years old were examined for a minimum of nine fatty acid oxidation disorders, using the global $\left[9,10-^{3} \mathrm{H}\right] \mathrm{myr}-$ istic acid oxidation assay in cultured fibroblasts from achilles tendon biopsies taken at postmortem examination.

Results-Three cases with fatty acid oxidation disorders and two carriers of the G985 mutation were found, all catagorised as non-SIDS or borderline SIDS. The global assay used has the advantage of simplicity.

Conclusions-These results indicate that disorders of fatty acid oxidation play a small but significant role in the cause of unexpected death in infants and young children, and that infants and children dying in this way should be regarded as high risk candidates for metabolic diseases.

(f Clin Pathol: Mol Pathol 1997;50:212-217)

Keywords: children; diagnosis; fatty acid oxidation disorders

In recent years the number of enzyme deficiencies in the fatty acid oxidation pathway recognised as giving rise to clinical disease has increased considerably. At least 14 disorders are now described, ${ }^{1-3}$ most of which are known to be responsible for cases of sudden and unexpected death in infancy. ${ }^{47}$ Medium chain acyl CoA dehydrogenase (MCAD) deficiency is the most common fatty acid oxidation disor- der and is inherited in an autosomal recessive $\vec{\omega}$ mode. The disorder is caused by mutations in the MCAD gene located on chromosome 1 . One prevalent mutation (G985) ${ }^{8}$ constitutes $90 \%$ of mutant alleles in diagnosed patients. ${ }^{9}$ In the majority of diagnosed cases, the disease $\vec{N}$ presents in infancy with hypoketotic hypogly- 윽 caemia and lethargy and is fatal in about $40 \%$ of cases, most often with the child dying during the first attack. ${ }^{210}$ Acute episodes are treated $\frac{0}{\infty}$ effectively with glucose, and long term therapy with frequent meals seems to prevent acute $\mathscr{\bullet}$ attacks. In northern Europe, the USA, and Australia the carrier frequency of the G985 point mutation is about one in 100 newborns. ${ }^{11-13}$ This is much higher than expected from the number of cases actually diagnosed in these countries, and it is estimated that in general only $10-20 \%$ of geneti- O cally disposed individuals are diagnosed. ${ }^{14}$ In Denmark, two new cases are expected each year, but only 10 cases have actually been diagnosed during the 20 year period since the first case of MCAD deficiency was identified. ${ }^{15}$ This $\frac{5}{3}$ might be explained partly by poor diagnostic efficiency and misdiagnoses. However, it is well known that asymptomatic but genotypically $\mathrm{O}$ affected siblings of patients with MCAD deficiency exist. ${ }^{16}$ It seems likely that geneti- Oㅡㅡㄹ cally defined MCAD deficiency is, in many cases, a condition that does not result in a serious clinical disease and, therefore, is not diagnosed. On the other hand several patients are known to have died in the first months of life due to MCAD deficiency, ${ }^{6} 1718$ and "missing cases" might also be hidden in this group.

A number of cases of MCAD deficiency who died during the first months of life have been misdiagnosed as SIDS. ${ }^{5619}$ Therefore, previ- $\stackrel{\Phi}{\Omega}$ ously, we investigated 120 well defined cases of $\stackrel{\mathbb{Q}}{\Omega}$ SIDS (who died from 1 January 1987 to 31 응 December 1988) to detect the most common 8 disease causing point mutation in the MCAD gene (G985). We used a highly specific

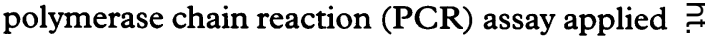
to Guthrie blood spots. ${ }^{20}$ In this strictly defined population we did not find over-representation of either homozygosity or heterozygosity for G985 compared with the frequency in the general population. ${ }^{21}$ Similar results have been found by other researchers. ${ }^{22-25}$ 
A major drawback of all these studies was that only a narrow group of child death (namely classic SIDS) was investigated, and that only one inborn error of fatty acid oxidation was looked for, namely MCAD deficiency due to the G985 mutation. Therefore, we broadened the scope of the investigation and present here the results from a prospective study investigating the role of fatty acid oxidation defects in unexpected death in infants and young children up to 4 years of age (who died from 1 June 1989 to 31 May 1992).

\section{Patients}

A presumed high risk group for fatty acid oxidation defects was selected, namely cases of sudden and unexpected death below 4 years of age. All cases underwent necropsy at the Institute of Forensic Medicine, University of Aarhus, from 1 June 1989 to 31 May 1992. The institute serves about two fifths of the Danish population (five million people). According to Danish law all cases of sudden and unexpected death are submitted to postmortem medicolegal investigation and in most cases of infant death a medicolegal necropsy is performed. The necropsy rate in Denmark is high and rather constant at around $92 \%$.

One hundred and twenty three cases of sudden and unexpected death below 4 years of age occurred in the study period of which only 12 were over 2 years of age. Achilles tendon biopsies were taken for establishment of fibroblast culture in 87 of the cases. The interval from death until the autopsy was performed varied from one to four days.

Study cases were classified into the following three groups ${ }^{26}{ }^{27}$ :

Classic SIDS-The sudden death of any infant or young child which is unexpected by history and in which a thorough postmortem examination fails to reveal an adequate cause of death. In short, deaths where the autopsy and clinical information do not reveal a cause of death and where the age range is between 7 and 365 days.

Borderline SIDS-Cases with non-lethal preexisting congenital disorders, clinical symptoms and/or postmortem findings not severe enough to explain the cause of death, still within the typical age range of SIDS (7-365 days).

Non-SIDS-Cases where the cause of death is explained by clinical information and/or the necropsy findings, and/or deaths not within the age interval of SIDS and borderline SIDS (7-365 days).

The classic SIDS group comprised 42 cases, with an age span from 0 to 9 months (mean 3.2). Nineteen cases were classified as borderline SIDS, aged from 0 to 7 months (mean 3.5). The non-SIDS group was 18 cases aged between 0 and 21 months (mean 8). The causes of death in the last group ranged from severe infections (especially respiratory) to heart and brain malformations, as well as a few abdominal catastrophies. A few died without apparent cause but these were outside the age range for classic SIDS (7-365 days).

\section{Methods}

FIBROBLAST CULTURES

Achilles tendon biopsies were removed aseptically during the postmortem examination and placed in pre-prepared sterile tubes containing $10 \mathrm{ml} \mathrm{RPMI}$ and $15 \%$ fetal calf serum, and standard doses of penicillin and streptomycin. The tubes were sealed and sent by special delivery without cooling. Further processing of the biopsies at the culture laboratory took place normally within a few hours after autopsy, but circumstances such as holidays and weekends could give delays of up to three days. In these cases, biopsies were kept at $4^{\circ} \mathrm{C}$ in culture medium. When processed the biopsies were cut initially into small pieces and then cultured in standard RPMI medium containing $15 \%$ fetal calf serum. Once cultures were established an aliquot of cells was frozen at $-120^{\circ} \mathrm{C}$.

GLOBAL FATTY ACID OXIDATION (B OXIDATION) ASSAY

The assay used in this study is a functional analysis of the fatty acid oxidation activity in cultured fibroblasts. ${ }^{28}$ The cells are incubated with $\left[9,10-{ }^{3} \mathrm{H}\right]$ myristic acid and the amount of liberated tritiated water is measured. If the fatty acid oxidation activity is reduced, lower amounts of tritiated water are liberated compared to control cells. Of the known fatty acid oxidation defects the following can be indicated/identified by the assay: cellular carnitine uptake defect, carnitine palmitoyl CoA transferase (CPT1) defect, carnitine palmitoyl CoA transferase (CPT2) defect, mitochondrial acylcarnitine/carnitine translocase defect, very long chain acyl CoA dehydrogenase (VLCAD) defect, medium chain acyl CoA dehydrogenase (MCAD) defect, trifunction protein (TPD) defect, electron transport flavoprotein (ETF) defect, and ETF dehydrogenase defect.

\section{URINE ANALYSIS}

In cases where urine was available, the profile of organic acids was analysed by a conventional gas chromatographic/mass spectrometric technique. In addition, a specific determination of selected fatty acid oxidation metabolites, comprising adipic acid, suberic acid, sebacic acid, hexanoyglycine, phenyl propionylglycine, and suberylglycine, was performed. ${ }^{29}$

G985 MUTATION ASSAY

The presence of the G985 mutation in DNA from the cultured fibroblasts was determined by a PCR based assay. ${ }^{30}$ The assay is based on the generation of a restriction endonuclease recognition site in the PCR product from the gene region when the template DNA contains the mutated allele. By including another restriction site in the antisense primer a control of the cleavage efficiency is obtained. ${ }^{20}$ After PCR and restriction enzyme digestion of the product, the resulting fragments are analysed by polyacrylamide gel electrophoresis (PAGE) and the presence of the mutated allele is indicated by the occurrence of a shorter band in the gel.

MCAD deficiency can be diagnosed unambiguously with this assay in approximately $80 \%$ 
Table 1 Fatty acid oxidation disorders and carriers of the G985 MCAD gene mutation found in the 79 cases of sudden and unexpected deaths. The results of the genetic counselling in the families of the positive cases are also shown

\begin{tabular}{lll}
\hline Disorder & Classification & Genetic counselling \\
\hline $\begin{array}{l}\text { Case 1 } \\
\text { A/A (985), reduced enzyme } \\
\text { activity }\end{array}$ & Borderline SIDS & $\begin{array}{l}\text { Younger brother with same } \\
\text { disorder diagnosed }\end{array}$ \\
$\begin{array}{l}\text { Case 2 } \\
\text { A/A (985), glutaric aciduria type } \\
\text { II (GAII) }\end{array}$ & Non-SIDS & $\begin{array}{l}\text { Older sibling has died } \\
\text { unexpectedly. Two younger } \\
\text { siblings found to be normal }\end{array}$ \\
$\begin{array}{l}\text { Case 3 } \\
\text { G985 (G/G) point mutation }\end{array}$ & $\begin{array}{l}\text { Non-SIDS, tentatively } \\
\text { diagnosed as Reye's } \\
\text { syndrome, based on fatty } \\
\text { infiltration in the liver }\end{array}$ & $\begin{array}{l}\text { New pregnancy, G985 G/G, } \\
\text { elective abortion }\end{array}$ \\
$\begin{array}{l}\text { Other } \\
\text { Two cases heterozygous for the } \\
\text { G985 A/G point mutation }\end{array}$ & Borderline SIDS & None given \\
\hline
\end{tabular}

of cases, namely those homozygous for the G985 mutation. A further $18 \%$ of MCAD deficiencies will carry one G985 mutation and, therefore, will also be indicated by the assay.

\section{Results}

Achilles tendon biopsies were obtained in 87 of the 123 cases of sudden and unexpected death autopsied during the three year period of investigation. Successful fibroblast cultures was obtained from 79 of the cases $(91 \%)$. This is a remarkably high success rate, considering that the biopsies were taken postmortem and there was sometimes a delay of up to four days before cultivation (from death to autopsy and from biopsy taking to further processing). Eight biopsies did not give rise to viable fibroblasts; in seven of these cases this was a result of severe uncontrollable infection. No explanation for failure of growth was found for the last biopsy. Urine was sampled postmortem from 21 of the 79 cases with successful fibroblast cultivation.

CASES WITH FATTY ACID OXIDATION DISORDERS

Three cases were identified with fatty acid oxidation defects. One case showed a reduced myristic acid oxidation rate, but with an undefined enzyme deficiency (case 1). A second case had multiple acyl CoA dehydrogenation defect (glutaric aciduria type II (GAII)), based on low myristic and palmitic acid oxidation rates and a typical GAII urine profile (case 2). The third case had MCAD deficiency, based on the presence of homozygosity for G985 (case 3). The results are summerised in table 1.

\section{Case 1}

A 4 month old boy, second child of healthy, unrelated white parents. The patient was born by normal delivery and developed normally for four months. He then developed a slight cold one day prior to his death. He was found lifeless in his bed in the morning and on arrival to the local hospital he was declared dead. Postmortem examination revealed nothing except slight pleural and pericardial petechial bleeding. Examination for pathological microorganisms was negative. The case was classified primarily as borderline SIDS, based on the clinical history. The standard screening programme revealed that he had the normal bases (A/A) at position 985 of the MCAD gene, but the tritium release assay for total fatty acid oxidation showed a diminished activity-about half the control value. When using $\left[9,10-{ }^{3} \mathrm{H}\right]$ myristic acid as substrate, an activity of $6.8 \mathrm{pmol} / \mathrm{min} / \mathrm{mg}$ protein was found (normal ? range 10.0-28.2); when using $\left[9,10-{ }^{3} \mathrm{H}\right]$ pal- 으 mitic acid a value of $6.9 \mathrm{pmol} / \mathrm{min} / \mathrm{mg}$ protein was found (normal range 9.9-26.1).

The parents had never exhibited symptoms $\vec{F}$ of metabolic disorders, and neither had the two $\overline{0}$ years older sister, who had developed normally. They were examined later for fatty acid oxida- $\frac{\bar{m}}{T}$ tion capacity in fibroblasts. All three were $\mathbb{\mathscr { D }}$ found to have activity within the normal range.

One year later the mother became pregnant $\vec{\circ}$ again. The parents wanted to have the baby even if it had the same condition as the index $\vec{\omega}$ patient, therefore, no prenatal diagnosis was performed. Immediately after delivery a blood sample and a skin biopsy were obtained from the child and at two months of age a urine $\vec{i}$ sample was collected. Examination of these $\vec{N}$ samples revealed that this child had the same 을 diminished fatty acid oxidation activity as the index case, and he excreted the detoxification product suberic acid marginally above the upper limit of normal, in spite of being asymptomatic. This child has since been on long term $\vec{\oplus}$ therapy with frequent meals.

Case 2

A 21 month old girl, third child of healthy parents from Kurdistan; the parents are second $\Phi$ cousins. The first child in the family died unexpectedly at the age of six months; the $\frac{3}{3}$ cause of death is unknown. The second child $?$ has developed normally. The third child, the index case, was born by normal delivery and had developed normally until the age of 21 을 months, when she developed varicella and fever 3 and after two days of illness she was found dead in bed. Postmortem examination revealed slight stasis and oedema of the lungs and nota- 을 ble fatty infiltration in the liver. The case was $D$ classified as non-SIDS due to the fulminant varicella infection and the age above 365 days. N It was possible to obtain a urine sample postmortem. The standard screening pro-. N gramme revealed $\mathrm{A} / \mathrm{A}$ at position 985 of the $\omega$ MCAD gene. The $3 \mathrm{H}$ release assay for total fatty acid oxidation revealed a value of $\frac{0}{\Phi}$ $2.7 \mathrm{pmol} / \mathrm{min} / \mathrm{mg}$ protein (normal range 10.028.2) with $\left[9,10-{ }^{3} \mathrm{H}\right]$ myristic acid as substrate, 0 and $5.0 \mathrm{pmol} / \mathrm{min} / \mathrm{mg}$ protein when using $\left[9,10-{ }^{3} \mathrm{H}\right]$ palmitic acid as substrate (normal $\stackrel{\oplus}{\oplus}$ range 9.9-26.1). Gas chromatography and $\stackrel{\mathbb{Q}}{\varrho}$ mass spectrometry analysis of the postmortem urine sample revealed a pattern consistent with $\delta$ the diagnosis of glutaric aciduria type II (GAII).

Later, fatty acid oxidation capacity and urine were examined from the parents and the older sister. All values were within the normal range. Since the birth of the index case the mother has given birth to two healthy children. They have had their fatty acid oxidation capacity measured and were found to be normal. 
Case 3

An 18 month old boy, first child of healthy unrelated parents. The boy died suddenly, after two days of diarrhoea and fever. The postmortem examination identified massive fatty infiltration in the liver, and oedema of the brain. The case was classified as non-SIDS with a tentative diagnosis of Reye's syndrome, based on the histological findings in the liver, even though no salicylates were found in the blood. Fatty acid oxidation measured with $\left[9,10-{ }^{3} \mathrm{H}\right]$ myristic acid as substrate was in the range found for patients homozygous for G985 $(2.6 \mathrm{pmol} / \mathrm{min} / \mathrm{mg}$ protein; normal range 10.0 28.2). The DNA analysis identified homozygosity for the mutation G985 (G/G).

Blood spots were obtained from the parents to test for the presence of the G985 mutation. Boths parents were found to be carriers. Later, the mother became pregnant again. In the 10th week a chorionic villus biopsy was taken. Isolated DNA from the fetus and DNA from the parents were analysed and demonstrated homozygosity for the G985 mutation in the fetus and confirmed the carrier status of both parents. The parents then chose to have the pregnancy terminated and DNA analysis on fetal tissues sampled after abortion confirmed the diagnosis. This case has been described previously. $^{31}$

CARRIERS OF G985

Two carriers of the G985 mutation were found among the 79 cases. In both, fatty acid oxidation was found to be within the normal range. No urine was available in either of the two cases. At autopsy, both cases had been classified as borderline SIDS.

\section{URINE ANALYSIS}

In 21 cases it was possible to obtain at least some urine. However, in 15 of these cases, including case 1 , the amounts of urine obtained were not sufficient to give any meaningful results. Of the six adequate urine samples five were normal. The sixth came from case 2 and exhibited a pattern indicative of GAII, with excretion of abnormal amounts of adipic acid, suberic acid, glutaric acid, ethylmalonic acid, and isovalerylglycine.

\section{Discussion}

The importance of fatty acid oxidation defects as a cause of death in infants who die suddenly and unexpectedly is still a matter of debate. There have been a growing number of reports documenting cases of fatty acid oxidation disorders with sudden death at an early age, especially MCAD deficiency, the most common fatty acid oxidation disorder in the general population..$^{53233}$ Such patients have often been classified as SIDS cases. The idea that MCAD deficiency might be a significant cause of SIDS prompted a number of researchers, including ourselves, to investigate the frequency of the common G985 MCAD gene mutation in SIDS cases. Invariably, the conclusion has been that MCAD deficiency does not contribute to SIDS, ${ }^{21-25} 3435$ especially when SIDS is defined strictly as cases where a thorough postmortem pathological examination fails to reveal an adequate cause of death. ${ }^{26}{ }^{27}$ The fact that the design of most of the previous studies automatically excludes all cases with a more serious clinical history prior to death, cases with positive pathological findings, and cases with an age below one week or above one year prompted us (together with a number of other workers) ${ }^{32} 33$ to examine a broader spectrum of child death.

The examination for fatty acid oxidation was performed on all unexpected deaths in childern below 4 years, who were autopsied during a three year period at the Institute of Forensic Medicine, University of Aarhus.

Seventy nine cases were examined and, as predicted, no fatty acid oxidation defects were detected in the 42 classical SIDS cases. However, one case with an uncharacterised fatty acid oxidation defect was found among the 19 borderline SIDS (case 1); in addition, one case of multiple acyl CoA dehydrogenation defect (case 2) and one case of MCAD deficiency (case 3) were identified among the 18 cases classified as non-SIDS.

The child that died in the setting of borderline SIDS (case 1) is interesting because he had only a slightly diminished fatty acid oxidation capacity. In spite of this, his death was caused only by a common cold, and autopsy revealed only slight pleural and pericardial petechial bleeding. The enzyme deficiency in this child has not been identified, but a later sibling showed, shortly after birth, a similar decreased myristic acid oxidation rate, and excreted slightly increased suberic acid in the urine. Unfortunately a diagnostically meaningful urine analysis was not available from the dead child. Increased urinary excretion of suberic acid, especially a suberic acid:adipic acid ratio greater than 1 , has been observed in children with MCAD deficiency during asymptomatic periods. ${ }^{30}$ In contrast to previous experience, where the increased suberic acid:adipic acid ratio was accompanied by increased excretion of hexanoylglycine, ${ }^{30}{ }^{36}$ the hexanoylglycine excretion in the sibling was not increased. Whether the two members of this family exhibit a long chain fatty acid defect, a generalised chain defect disorder, or perhaps a "mild" MCAD deficiency caused by mutations other than G985, which under certain nutritional and metabolic conditions can result in a fulmination metabolic decompensation, is a subject for future studies. That the latter is a possibility is indicated by two previously published compound heterozygous patients, who harbour the G985 MCAD mutation on one allele and another mutation (T157) on the other. ${ }^{6}$ In one child who was initially classified as a SIDS case, this combination of mutations resulted in death at 3 days of age; in the other child the same combination of mutations gave rise to a milder disease course, and a myristic acid oxidation rate in cultured fibroblasts comparable to the one seen in the present child (4.9v $6.8 \mathrm{pmol} / \mathrm{min} / \mathrm{mg}$ protein, respectively). ${ }^{6}$

The two other cases found in this study with MCAD deficiency and multiple acyl CoA 
dehydrogenation defect, respectively, were clasified as non-SIDS.

The child with multiple acyl CoA dehydrogenation defect (case 2), who died at 21 months old is more problematical. Usually, the fatal cases of GAII occur early in life, but cases dying later in life are known. ${ }^{37}$ Furthermore, only some of the clinical and pathological findings usually present in GAII patients were seen. However, the massive excretion of adipic and suberic acid, with smaller but greatly elevated excretion of glutaric acid, ethylmalonic acid, and isovalerylglycine, together with low myristic and palmitic acid oxidation rates, unambiguously identified the enzyme deficiency to be multiple and compatible with the picture seen in GAII.

The MCAD deficient child (case 3) was diagnosed initially as having Reye's syndrome and revealed clinical and pathological findings compatible with the current knowledge about MCAD deficiency including fever before death, a massive fatty liver, and oedema of the brain at necropsy. ${ }^{38}$ Molecular analysis showed homozygosity for the G985 MCAD gene mutation, which was confirmed by the detection of the mutation in its heterozygous form in both parents. This case adds credence to the concept that the common G985 mutation in MCAD deficiency can be a very serious disease.

Although the number of investigated cases in this study is not very large it is striking that: no individuals with fatty acid oxidation defect were found in the cases classified as SIDS; the "mild" case was found in the group of borderline SIDS cases; and the two cases of identifiable fatty acid oxidation defects were found among 18 cases of non-SIDS. This underlines the importance of thorough clinical, pathological, and biochemical investigation, especially of non-SIDS death in childhood, in order to identify fatty acid oxidation defects.

The validity of the high frequency of identified cases in the present investigation is supported by two reports that were published when this work was in progress. Bennett and Powell investigated 58 infants from rural areas of Illinois, who were below 2 years of age when they died suddenly and unexpectedly with no significant medical history. ${ }^{33}$ This seems to be a group of cases comparable to the one investigated in this study, except for the age span. Urine, bladder wall swabs, and/or blood were examined from the dead children, and four cases of indicated fatty acid oxidation defects were found, in addition to two cases of possible respiratory chain deficiencies. Cells from the dead children were not investigated, but the authors point out that such measurements of fatty acid oxidation rates would have improved the protocol, and further characterised the cases. The assay of fatty acid oxidation as part of the investigation would probably also improve the diagnostic sensitivity but, as Bennett and Powell point out, the additional costs would be prohibitive for the study. ${ }^{33}$ It would have been interesting to see in the present study whether all three cases of fatty acid oxidation defects found would have exhib- ited pathological urinary metabolite profiles. Only urine from the GAII case was available, and gave a diagnostic profile. It is, however, our contention that we would have detected the MCAD deficient case easily by urinalysis, had sufficient urine been available. The first case 으 would probably also have been detected, since a slight elevation of suberic acid was detected $\stackrel{0}{\overrightarrow{7}}$ in the urine of an asymptomatic sibling. However, until the nature of the first case has been $\stackrel{\vec{F}}{\rightarrow}$ elucidated, the possibility exists that such a 듬 case would be missed by ordinary metabolic $\frac{\text { 음 }}{\bar{N}}$ profile analysis by gas chromatography, be- $\frac{\vec{D}}{2}$ cause slight elevations of the metabolites would $\cong$ be easily overlooked. It could well be that Ben- के net and Powell would have detected more than $\overrightarrow{0}$ four cases of fatty acid oxidation defects if they $\vec{\overrightarrow{ }}$ had used a fibroblast fatty acid oxidation assay. The same may be true for another investigation $\frac{3}{3}$ of pathological metabolites in frozen liver sam- 웅 ples from 100 dead children from Maryland ${ }^{32}$; O the definition used by the clinicians for the classification of SIDS is not clear, but it is $\vec{N}$ striking that in as many as 81 of the cases the 을 death certificates gave SIDS as the cause of death. Even if the classification of the cases is less restricted than ours and the number of non-SIDS cases greater than 19, the finding of $\stackrel{\oplus}{\hookrightarrow}$ five indicated fatty acid oxidation defects con- $\overrightarrow{0}$ stitutes a high frequency, comparable to that found in the present study and that by Bennett and Powell. ${ }^{33}$

In conclusion, these three studies show that when concentrating on groups diagnosed as borderline and non-SIDS (as classified in the $\frac{\mathbb{Q}}{2}$ present study), the frequency of fatty acid oxi- $\overrightarrow{\vec{F}}$ dation defects is high. This high frequency is found irrespective of whether fibroblast cultures are used to measure the fatty acid oxidation rate or careful analysis of metabolic profiles in liver, urine, or blood are performed. However, with regard to urine, it is our $\frac{5}{3}$ experience that it is difficult to obtain sufficient urine from small children at autopsy to get meaningful profile data. Similarly, our success rate with collection of useful blood samples postmortem is very low. Extraction and evaluation of metabolites from liver tissue is not complicated, but demands some expertise, while o growing fibroblast cultures is cumbersome and expensive.

The best method will probably be a matter of personal preference and institutional conven- 0 tion. However, we find it beneficial to use the global fatty acid oxidation method, at least if the selected group is not too large, in order to ensure the highest possible success rate and because the assay can be performed on most cases, with a good opportunity to perform additional assays when a fatty acid oxidation defect is indicated.

Even if the number of fatty acid oxidation defects does not make up more than a few percent of the total cases of sudden death in childhood, including the SIDS, it is important to identify inherited causes, such as the fatty acid oxidation defects, because genetic counselling can identify other members of the family who are in a group at high risk of dying suddenly and unexpectedly. Practising pathologists 
should keep fatty acid oxidation disorders in mind when dealing with sudden and unexpected death in childhood, especially if there is no clinical history or pathological findings indicating the cause of death. This is even more so if the clinical history and pathological findings show a picture of banalities such as cold, diarrhoea, varicella, and so on. As mentioned, whether urine, bladder wall swabs, blood, frozen liver samples, or fibroblast cultivation is used is a matter of personal preference and institutional convention, but the possibility of genetic counselling should be available.

This work was supported by the foundations of The University of Aarhus, Dagmar Marshall, Hartmann Brothers, Grosserer LF Foght, and King Christian IX.

1 Gregersen N, Andresen BS, Bross P, Bolund L, Kølvraa S. Disorders of mitochondrial fatty acid oxidation-especially medium-chain acyl CoA dehydrogenase (MCAD) deficiency. In: Farriaux JP, Dhondt JL, eds. New horizons in neonatal screening. Amsterdam: Elsevier, 1995:247-55.

2 Roe CR, Coates PM. Mitochondrial fatty acid disorders. In: Scriver CR, Beudet AL, Sly WS, Valle D, eds. The metabolic and molecular basis of inherited disease. New York: McGrawHill, 1995:1501-33.

3 Aoyama T, Souri M, Ushikubo S, Kamijo T, Yamaguchi S, Kelley RI, et al. Purification of human very-long-chain acyl-coenzyme A dehydrogenase $\mathrm{A}$ and characterization of its deficiency in seven patients. $\mathcal{F}$ Clin Invest 1995;95:246573.

4 Harpey JP, Charpentier C, Paturneau-Jouas M. Sudden infant death and inherited disorders of fatty acid betaoxidation. Biol Neonate 1990;58(Suppl):70-80.

5 Politt RJ. Defects in mitochondrial fatty oxidation: clinical presentations and their role in sudden infant death. Paedi presentations and their
atr Paedol 1993;28:13-7.

6 Andresen BS, Bross P, Winter V, Kølvraa S, Jensen T, Bolund $\mathrm{L}$, et al. A rare disease-causing mutation in the medium-chain acyl CoA dehydrogenase (MCAD) gene changes a conserved arginine, previously shown to be functionally essential in short-chain acyl-CoA dehydrogenase (SCAD). Am ₹ Hum Genet 1993;53:730-9.

7 Brackett JC, Sims HF, Steiner RD, Nunge M, Zimmermann $\mathrm{EM}$, deMartinville $\mathrm{B}$, et al. A novel mutation in medium-chain acyl-CoA dehydrogenase causes sudden medium-chain acyl-CoA dehydrogenase causes

8 Tanaka K, Yokota I, Coates PM, Strauss AW, Kelly DP, Zhang $\mathrm{Z}$, et al. Mutation in the medium-chain acyl-CoA dehydrogenase (MCAD) gene. Hum Mutation 1992;1:271 9.

9 Mutations causing medium-chain acyl-CoA dehydrogenase deficiency: a collaborative compilation of data from 172 patients. In: Coates PM, Tanaka K, eds. New development in fatty oxidation. New York: Wiley-Liss, 1992:499-506.

10 Iafolla AK, Thompson RJ, Roe CR. Medium-chain acyl-CoA dehydrogenase deficiency: clinical course in 120 acyl-CoA dehydrogenase deficiency: clinical

11 Blakemore AIF, Singleton H, Politt R, Engel PC, Kølvraa S, Gregersen $\mathrm{N}$, et al. The frequency of the G985 MCAD mutation in the general population. Lancet 1991;337:298 9.

12 Gregersen N, Winter V, Curtis D, Deufel T, Willems P, Ponzone A. et al. Medium-chain acyl-CoA dehydrogenase (MCAD) deficiency: the prevalent mutation G985 (K304E) is subject to a strong founder effect from northwestern Europe. Hum Hered 1993;43:342-50.

13 Matsubara Y, Narisawa K, Tada K, Ikeda H, Ye-qi Y, Danks $\mathrm{DM}$, et al. Prevalence of K329E mutation in medium-chain acyl-CoA dehydrogenase gene determined from Guthrie cards. Lancet 1991;358:552-3.

14 Gregersen N, Winter V, Kølvraa S, Andresen BS, Bross P, Blakemore AIF, et al. Molecular analysis of medium-chain acyl-CoA dehydrogenase deficiency: a diagnostic approach. In: Coates PM, Tanaka K, eds. New development in fatty oxidation. New York: Wiley-Liss, 1992:441-52.

15 Gregersen N, Lauritzen R, Rasmussen K. Suberylglycine excretion in the urine of a patient with dicarboxylic excretion in the urine of a patient with

16 Duran M, Hofkamp M, Rhead WJ, Saudubray JM, Wadman SK. Sudden child death and "healthy" affected family members with medium-chain acyl-CoA dehydrogenase deficiency. Pediatrics 1986;78:1052-7.
17 Nobukuni Y, Yokoo T, Ohtani Y, Endo F, Aoki S, Yoshinaga M. et al. Neonatal onset of medium-chain acyl-CoA dehydrogenase deficiency in two siblings. Brain Dev 1988;10 129-34.

18 Catzeflis C, Bachmann C, Hale DE, Coates PM, Wiesmann $\mathrm{U}$, Colombo JP, et al. Early diagnosis and treatment of neonatal medium-chain acyl-CoA dehydrogenase deficiency: report of two siblings. Eur F Pediatr 1990;149:577-81.

19 Bennett MJ, Allison F, Politt RJ, Variend S. Fatty acid oxidation defects as cause of unexpected death in infancy. In: Tanaka K, Coates, PM, eds. Fatty oxidation: clinical, biochemical and molecular aspects. New York: Alan R Liss, 1990:349-64

20 Gregersen N, Blakemore AIF, Winter V, Andresen BS, Kolvraa S, Bolund L, et al. Specific diagnosis of medium-chain acyl-CoA dehydrogenase (MCAD) deficiency in dried blood spots by a polymerase chain reaction (PCR) assay detecting a point-mutation (G985) in the MCAD gene. Clin Chim Acta 1991;203:23-4.

21 Lundemose JB, Gregersen N, Kølvraa S, Petersen BN, Gregersen M, Helweg-Larsen $\mathrm{K}$, et al. The frequenzy of a disease-causing point-mutation in the gene coding for medium-chain acyl-CoA dehydrogenase (MCAD) in sudden infant death syndrome (SIDS). Acta Paediatr 1993; 82:544-6.

22 Holten JB, Allen JT, Green CA, Partington S, Gilbert RE, Berry PJ. Inherited metabolic diseases in sudden infant death syndrome. Arch Dis Child 1991;66:1315-7.

23 Miller ME, Brooks JG, Forbes N, Insel R. Frequency of medium-chain acyl-CoA dehydrogenase deficiency G-985 mutation in sudden infant death syndrome. Pediatr Res 1992;31:305-7.

24 Chinsky J, Tolsma T, Cowan T, Blitzer M. MCAD deficiency in SIDS. Am f Hum Gen 1991;49(Suppl):A183.

25 Arens R, Gozal D, Jain K, Muscati S, Heuser ET, Williams JC, et al. Prevalence of medium-chain acyl-CoA dehydrogenase deficiency in sudden infant death syndrome. 7 Pediatr 1993;122:715-8.

26 Bergman AB, Berkwith JB, Ray CC. Sudden infant death syndrome. Seattle: University of Washington Press, 1970:17-8.

27 Gregersen M, Rajs J, Laursen H, Baandrup U, Frederiksen $\mathrm{P}$, Gidlund E, et al. Pathologic criteria for the Nordic study of SIDS. In: Rognum TO, ed. Sudden infant death syndrome, new trends in the nineties. Oslo: University Press, 1995:50-8.

28 Manning NJ, Olpin SE, Politt RJ, Webley J. A comparison of $[9,10-3 \mathrm{H}]$ myristic acids for the detection of defects of fatty acid oxidation in intact cultured fibroblasts. 7 Inher Metab Dis 1990;13:58-68.

29 Gregerson N, Winter V, Lyonnet S, Saududray JM, Wendel $\mathrm{U}$, Jensen TG, et al. Molecular characterization and urinary excretion pattern of metabolites in two families with MCAD deficiency due to compound heterozygocity with a 13 base-pair insertion on one allele. F Inher Metab Dis 1994;17:169-84.

30 Kwok S, Kelogg DE, McKinney N, Spasic D, Goda L, Levenson $\mathrm{C}$, et al. Effect of primer-template mismatches on polymerase chain reaction. Human immunodeficiency polymerase chain reaction. Human immunodeficiency virus 1005 .

31 Gregersen N, Winter V, Jensen PKA, Kølvraa S, Andresen $\mathrm{BS}$, Bross $\mathrm{P}$, et al. Prenatal diagnosis of medium-chain acyl CoA dehydrogenase (MCAD) deficiency in a family with a fatal case of sudden unexpected death in childhood. Prenatal Diagn 1995;15:82-6.

32 Boles RG, Martin SK, Blitzer MG, Rinaldo P. Biochemical diagnosis of fatty acid oxidation disorders by metabolite analysis of postmortem liver. Hum Pathol 1994;25:735-41.

33 Bennett MJ, Powel S. Metabolic disease and sudden, unexpected death in infancy. Hum Pathol 1994;25:742-6.

34 Miller M, Brooks J, Forbes N, Insel R. Frequency of G-985 mutation in medium-chain acyl-CoA dehydrogenase (MCAD) deficiency in sudden infant death syndrome. Prog Clin Biol Res 1992;375:495-8.

35 Lemieux B, Giguerre R, Cyr D, Shapcott D, McCann M, Tuchman $M$. Screening urine of 3-week-old newborns: lack of association between sudden infant death syndrome and some metabolic disorders. Pediatrics 1993;85:986-8.

36 Gregersen N, Kolvraa S, Rasmussen K, Mortensen PB, Divry P, David M, et al. General (medium-chain) acyl-CoA dehydrogenase deficiency (non-ketotic dicarboxylic aciduria): quantitative urinary excretion pattern of 23 biological significant organic acids in 3 cases. Clin Chim Acto 1983;132:181-91.

37 Frerman FE, Goodman SI. Nuclear encoded defect of mitochondrial respiratory chain, including glutaric acidemia type II. In: Scriver CR, Beudet AL, Sly WS, Valle $\mathrm{D}$, eds. The metabolic and molecular basis of inherited disease. Dew York: McGraw Hill, 1995:1611-30.

38 Bonell HJ, Beckwith JB. Fatty liver in sudden childhood death: implications for Reye's syndrome? Am f Dis Child 1986;140:30-3. 